\title{
The efficacy and safety of oncolytic viruses in the treatment of intermediate to advanced solid tumors: a systematic review and meta-analysis
}

\author{
Peng Gao, Guanxiong Ding, Lujia Wang \\ Department of Urology, Huashan Hospital, Fudan University, Shanghai, China \\ Contributions: (I) Conception and design: P Gao; (II) Administrative support: P Gao; (III) Provision of study materials or patients: All authors; (IV) \\ Collection and assembly of data: G Ding, L Wang; (V) Data analysis and interpretation: P Gao; (VI) Manuscript writing: All authors; (VII) Final \\ approval of manuscript: All authors. \\ Correspondence to: Peng Gao. Department of Urology, Huashan Hospital, Fudan University, 12 Urumqi Middle Road, Jing'an District, Shanghai \\ 200000, China. Email: gaopeng200976@yeah.net.
}

Background: Cancer treatment remains one of the most formidable challenges worldwide. Some novel treatment strategies, including molecularly targeted therapy, gene therapy, and cellular immunotherapy, have also been investigated to improve therapeutic effects for cancer patients and have demonstrated unexpected positive effects. This systematic review and meta-analysis evaluated the efficacy and safety of oncolytic virus (OV) monotherapy or combination therapy for intermediate to advanced solid tumors.

Methods: We retrieved articles from PubMed, Embase, Web of Science, CNKI, Wanfang and VIP. The quality of the included studies was assessed by Review Manager Software version 5.3. STATA software was used to perform meta-analyses of efficacy, overall survival (OS) and adverse reactions.

Results: A total of 22 studies involving 3,996 patients were included in this analysis, including 13 H101 studies, 5 T-VEC studies, 2 Pexa-Vec studies, 1 HF10 study and 1 Reolysin study. Regarding oncolytic adenovirus H101, meta-analysis showed that patients treated with H101 monotherapy or H101 combined with chemotherapy had a significantly higher objective response rate (ORR) than those treated with chemotherapy. Patients in the H101 and T-VEC groups had significantly longer effect size (ES) than the control group patients. The odds ratio (OR) and ES of patients with hepatocellular carcinoma, lung cancer and melanoma treated with OV were analyzed. For the safety profile, the total incidence of adverse reactions was similar in both groups. In terms of the other OVs, according to a systematic review, we found that after Reolysin treatment, the ORR was $26.9 \%$ in patients with head and neck cancer. The phase I study of HF10 exhibited some therapeutic potential. The adverse events (AEs) associated with the other OVs mainly included fever, nausea and vomiting, leukopenia, and hypotension.

Discussion: OVs are effective and well tolerated for the treatment of intermediate to advanced solid cancer and represent a promising therapeutic approach for solid cancers.

Keywords: Oncolytic virus (OV); intermediate to advanced solid tumor; efficacy; safety; systematic review; metaanalysis

Submitted May 25, 2021. Accepted for publication Aug 04, 2021.

doi: $10.21037 /$ tcr-21-905

View this article at: https://dx.doi.org/10.21037/tcr-21-905 


\section{Introduction}

Cancer is the second leading cause of death following heart disease (1). Cancer treatment remains one of the most formidable challenges worldwide. Some novel treatment strategies with multiple mechanisms of action (MOAs), including molecularly targeted therapy, gene therapy, and cellular immunotherapy, have also been investigated to improve therapeutic effects for cancer patients and have demonstrated some unexpected positive effects (2). Oncolytic virus $(\mathrm{OV})$ therapy is a novel and promising approach for tumor immunotherapy (3) that appears to have a wide spectrum of anticancer activity with minimal human toxicity.

OVs are used in their natural state or genetically modified to enhance the selectivity of the viruses for cancer cells and reduce virulence to normal cells without integrating into host cellular chromosomes. Hence, OVs can selectively replicate in cancer cells and lyse them, and then the viral infection spreads to and kills surrounding tumor cells, eventually leading to a reduction in the tumor volume (3). Different types of OVs have certain limitations in the treatment of tumors. First, the replication ability of such viruses is limited. OVs can also induce systemic antitumor immunity (cancer vaccine properties) (4) by enhancing antigen release/recognition and subsequent immune activation. OVs are generated from two distinct mechanisms: selective replication in tumor cells, resulting in a direct lytic effect on tumor cells, and induction of systemic antitumor immunity. OVs kill tumor cells and release tumor-associated antigens, viral pathogen-associated molecular pattern signals, cellular danger-associated molecular pattern signals, and cytokines. These molecules promote the maturation of antigen-presenting cells and active effector $\mathrm{T}$ cells, which mediate antitumor immunity upon antigen recognition (5).

Currently, OVs include adenovirus (Ad), herpes simplex virus (HSV), Newcastle disease virus (NDV), measles virus, reovirus, parvovirus, etc., which have been investigated in numerous preclinical or clinical settings (3). Despite the numerous viruses investigated, some OVs have not been approved for clinical use worldwide. For example, reovirus oncolysis has been linked to activation of RAS mutations with inactivation of double-strand RNA-activated protein kinase, thereby promoting reovirus replication (6). PexaVec (pexastimogene devacirepvec; JX-594) is a thymidine kinase gene-inactivated oncolytic vaccinia virus that was well tolerated in liver cancers via intratumoral injection in phase $1 / 2$ trials (7).
Presently, only three kinds of OVs have been approved for clinical use worldwide, including H101 (Oncorine $^{\circledR}$ ), talimogene laherparepvec (Imlygic, T-VEC) and Rigvir H101. In 2005, the State Food and Drug Administration of China approved H101 for the treatment of advanced nasopharyngeal carcinoma (8). T-VEC is a recombinant HSV expressing human granulocyte-macrophage colony stimulating factor (GM-CSF) (9). T-VEC has been approved by the US Food and Drug Administration for the treatment of melanoma, and although a phase I clinical trial in liver cancer has been carried, research on other solid tumors has not yet been conducted. Rigvir is an OV belonging to the Picornaviridae family, Enterovirus genus, ECHO group, type 7 , that has not been genetically modified but has been selected and adapted for melanoma (10). In a study involving gastric cancer patients, an increase of up to $20 \%$ in T lymphocytes in the tumor and mucous was observed after Rigvir administration before surgery in earlystage gastric cancer patients.

Although OVs have demonstrated a number of positive therapeutic features in solid tumors over the past 15 years, the efficacy and safety of OVs in intermediate to advanced solid tumors remain controversial (11). This systematic review combined related randomized controlled trials (RCTs) and retrospective studies to evaluate the efficacy and safety of OVs in patients with solid tumors. We present the following article in accordance with the PRISMA reporting checklist (available at https://dx.doi.org/10.21037/tcr-21-905).

\section{Methods}

\section{Literature search strategy}

The two authors (Peng Gao and Guanxiong Ding) independently performed study selection to identify articles from PubMed, Embase, Web of Science, CNKI, Wanfang and VIP. We systematically searched these databases to identify all relevant studies published in English or Chinese up to June 19, 2020. RCTs or retrospective articles of OVs in intermediate and advanced tumors were collected. The following search terms were used: 'oncolytic virus H101', 'oncolytic virus', 'oncolytic poxvirus', 'solid tumor', 'herpes simplex virus', 'reovirus, vaccine virus', 'newcastle disease virus' 'Oncorine', 'recombinant human adenovirus type 5' 'oncolytic adenovirus H101', 'HF10', 'Pexa-Vec', 'Reolysin', 'Talimogene Laherparepvec' and 'T-VEC'. Obviously irrelevant articles were excluded by reading the titles and abstracts to conduct preliminary screening. The authors 
evaluated the secondary selection of literature for inclusion by carefully reading the whole texts. Any disagreement was resolved by a third reviewer. Sensitivity analyses were performed to examine robustness by removing individual studies one at a time.

\section{Study selection}

The studies met the following criteria: (I) patients were diagnosed with intermediate to advanced solid tumors, regardless of nationality, sex or race; (II) the study design was an RCT, retrospective study or clinical study; (III) OVs were administered as monotherapy or combined with chemotherapy; and (IV) the outcome measures contained at least one of the following criteria: the objective response rate (ORR), complete response (CR), partial response (PR), stable disease (SD), progressive disease (PD), overall survival (OS), progression-free survival (PFS), disease control rate (DCR), time to progression (TTP) and safety. Studies were excluded if they met the following criteria: (I) unpublished literature; (II) studies without OV administration; (III) the study design was not rigorous (e.g., the criteria for outcome measures were not standardized, the sample data were not explained clearly or completely); (IV) the studies were not in Chinese or English; and (V) duplicate data.

\section{Data extraction}

Data were extracted from the included articles by the authors independently. The following information was obtained from each study: the author, publication year, participants' details (the number of patients enrolled, participant demographics, cancer types), intervention measures, outcomes, and adverse events (AEs).

\section{Quality assessment}

The quality of the included articles was evaluated with the Cochrane Risk of Bias tool (version 5.1.0, updated in March 2011), which consists of seven evaluation domains, including 'random sequence generation', 'allocation concealment', 'blinding of participants', 'blinding of key personnel', 'incomplete outcome data', 'selective outcome reporting' and 'other sources of bias'. Each assessment was divided into three levels: low risk, unclear risk and high risk.

\section{Statistical analysis}

The meta-analysis was conducted with STATA 15.0 software. For continuous variables, we extracted the mean and standard deviation to calculate the weighted mean deviation and $95 \%$ CI. For binary variables, we extracted the OR or HR with the corresponding $95 \%$ CI. Heterogeneity was assessed with the chi-square $\left(\chi^{2}\right)$ test and the index of heterogeneity $\left(\mathrm{I}^{2}\right)$ statistic at an alpha level of 0.10 . Moderate to substantial heterogeneity was identified if $\mathrm{P} \leq 0.1$ or $\mathrm{I}^{2} \geq 50 \%$, which warranted a random effects model. If no interstudy heterogeneity was found with $\mathrm{P}>0.1$ or $\mathrm{I}^{2}<50 \%$, we used a fixed effects model. Subgroup analysis was performed according to the possible heterogeneous factors, and sensitivity analysis was also performed to analyze the stability of the meta-analysis.

\section{Results}

\section{Identification and selection study}

First, a total of 31,867 articles were obtained by using the retrieval strategy. Then, 31,828 studies were excluded after title and abstract evaluations. After reading the full texts, 23 articles were eliminated due to incomplete or duplicate data, and 22 articles were finally included. The study selection procedures were performed in accordance with the PRISMA flowchart (Figure 1).

\section{Characteristics of the included studies and quality assessment}

A total of 3,996 patients were included in the 22 articles (7,12-32), including 7 articles $(7,12-17)$ on hepatocellular carcinoma (HCC), 5 articles (18-22) on lung cancer, 5 articles on melanoma $(23-27), 2$ studies $(28,29)$ on pancreatic cancer, 1 article (30) on head and neck cancer, 1 study (31) on esophageal cancer, and 1 study (32) was on cancer. Five types of OVs (H101, HF10, Pexa-Vec, Reolysin and T-VEC) were used among the included studies. The characteristics of the included studies are shown in Table 1. The included articles contained 15 RCTs and 7 retrospective studies. Most RCTs reported randomized methods, and a few studies used blinded methods and allocation concealment. The assessment of the risk of bias in the included studies is shown in Figure 2. 


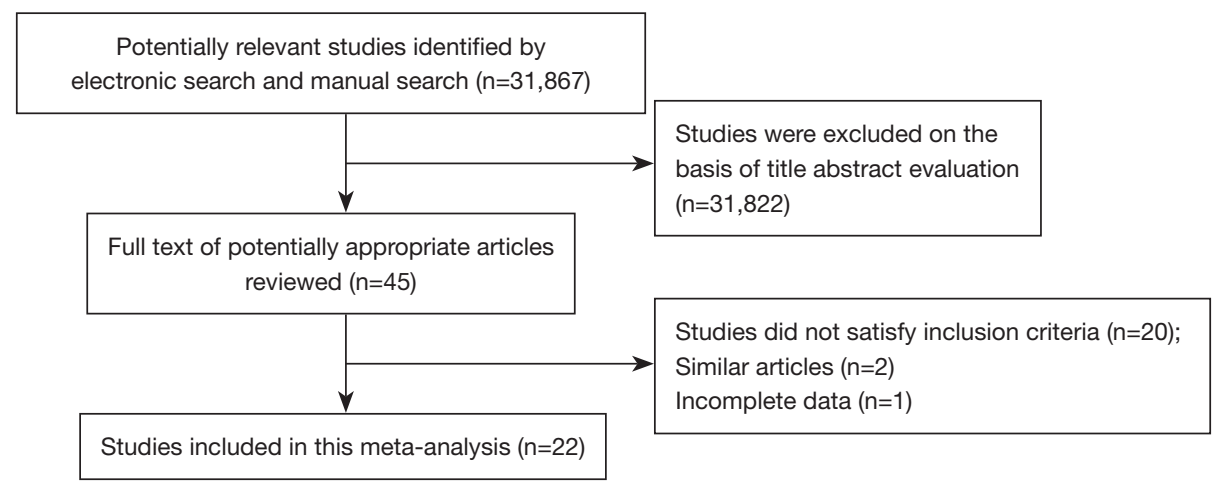

Figure 1 Flow diagram of the literature search and study selection.

\section{Efficacy}

A total of 12 articles $(12,14-16,18-22,28,31,32)$ reported the ORR of H101 in the treatment of solid tumors, such as HCC and lung cancer, 5 of which $(19-22,28)$ compared H101 monotherapy versus chemotherapy, while 7 articles (12,14-16,18,31,32) compared H101 combined with chemotherapy versus chemotherapy alone. The fixed effects model showed that the ORR in patients treated with $\mathrm{H} 101$ was significantly higher than that in patients treated with chemotherapy $[\mathrm{OR}=1.94,95 \% \mathrm{CI}$ : (1.61, 2.33), $\mathrm{P}<0.001$ ], without heterogeneity among articles $\left(\mathrm{P}=0.536, \mathrm{I}^{2}=0\right)$ (Figure $\left.3 A\right)$. Subgroup analysis showed that treatment with H101 monotherapy or H101 combined with chemotherapy was more effective $[\mathrm{OR}=2.99,95 \%$ CI: $(1.79,4.97), \mathrm{P}<0.001]$ [OR $=1.81,95 \%$ CI: $(1.49,2.21)$, $\mathrm{P}<0.001]$ than chemotherapy alone. In addition, this study also showed that T-VEC had a significant therapeutic effect on melanoma $[\mathrm{OR}=6.73,95 \% \mathrm{CI}$ : $(3.52,12.84), \mathrm{P}<0.001]$ (Figure 3B).

Combined with funnel plots and Egger's test, publication bias was identified among the included studies as a whole, whereas no publication bias was found among the combined treatment studies $(\mathrm{P}>0.05)$ or among the monotherapy studies $(\mathrm{P}<0.05)$ (Figure 4$)$. Pooled analysis of 4 articles $(13,14,18,31)$ showed the OS rates between the H101 group and chemotherapy group. The results of the fixed effects model indicated that compared with that for the chemotherapy group, the median survival ratio of the experimental group to the control group was estimated to be $1.16(0.83,1.61)$ (Figure $5 A)$. The median survival ratio point estimate and $95 \%$ CI for OS obtained from both effect models were $1.23(1.15,1.31)$, indicating that the median survival in the intervention group was 1.23 times that in the control group (Figure 5B). Furthermore, we analyzed the therapeutic effects of H101 on different types of tumors and found that the OR of H101 for liver cancer was the lowest; that is, the therapeutic effect was the least obvious (Figure 6). At the same time, the results showed that T-VEC had the most obvious therapeutic effect on melanoma.

\section{Safety}

A total of 7 articles reported the adverse reactions in patients treated with H101 in detail $(15,16,18,19,22,28,31)$. Pooled analysis of the random effects model identified that the overall incidence rate of treatment-related adverse effects was similar between the H101 group and the control group [OR $=1.20,95 \% \mathrm{CI}:(0.91,1.59), \mathrm{P}>0.05]$, and heterogeneity existed among these studies $\left(\mathrm{I}^{2}=71.8 \%, \mathrm{P}=0.001\right)$. Subgroup analysis showed that compared with that in the chemotherapy group, only the incidence rate of fever was higher in the H101 group [OR $=3.84,95 \%$ CI: $(1.44,10.24)$, $\mathrm{P}<0.05$ ], and the incidence rates of other adverse reactions, such as gastrointestinal reaction [OR $=1.11,95 \% \mathrm{CI}$ : (0.76, $1.61), \mathrm{P}>0.05]$, leucopenia $[\mathrm{OR}=0.85,95 \% \mathrm{CI}:(0.55,1.32)$, $\mathrm{P}>0.05]$ and myelosuppression $[\mathrm{OR}=0.64,95 \% \mathrm{CI}:(0.26$, 1.61), $\mathrm{P}>0.05$ ], were similar (Figure 7).

In terms of the other OVs, after treatment with Pexa-Vec in liver cancer, the most frequent minor complications were fever (8\%) and hypotension (8\%) (17). Using Reolysin for head and neck cancer, the most frequent adverse reactions were influenza-like symptoms (such as chills, fatigue, myodynia and fever), and others were neutropenia (16.1\%), asymptomatic lymphopenia (6.5\%) and anemia (3.2\%) (30) (Table 2). The most common AEs with T-VEC were fatigue, 
Table 1 The basic characteristics of the included studies

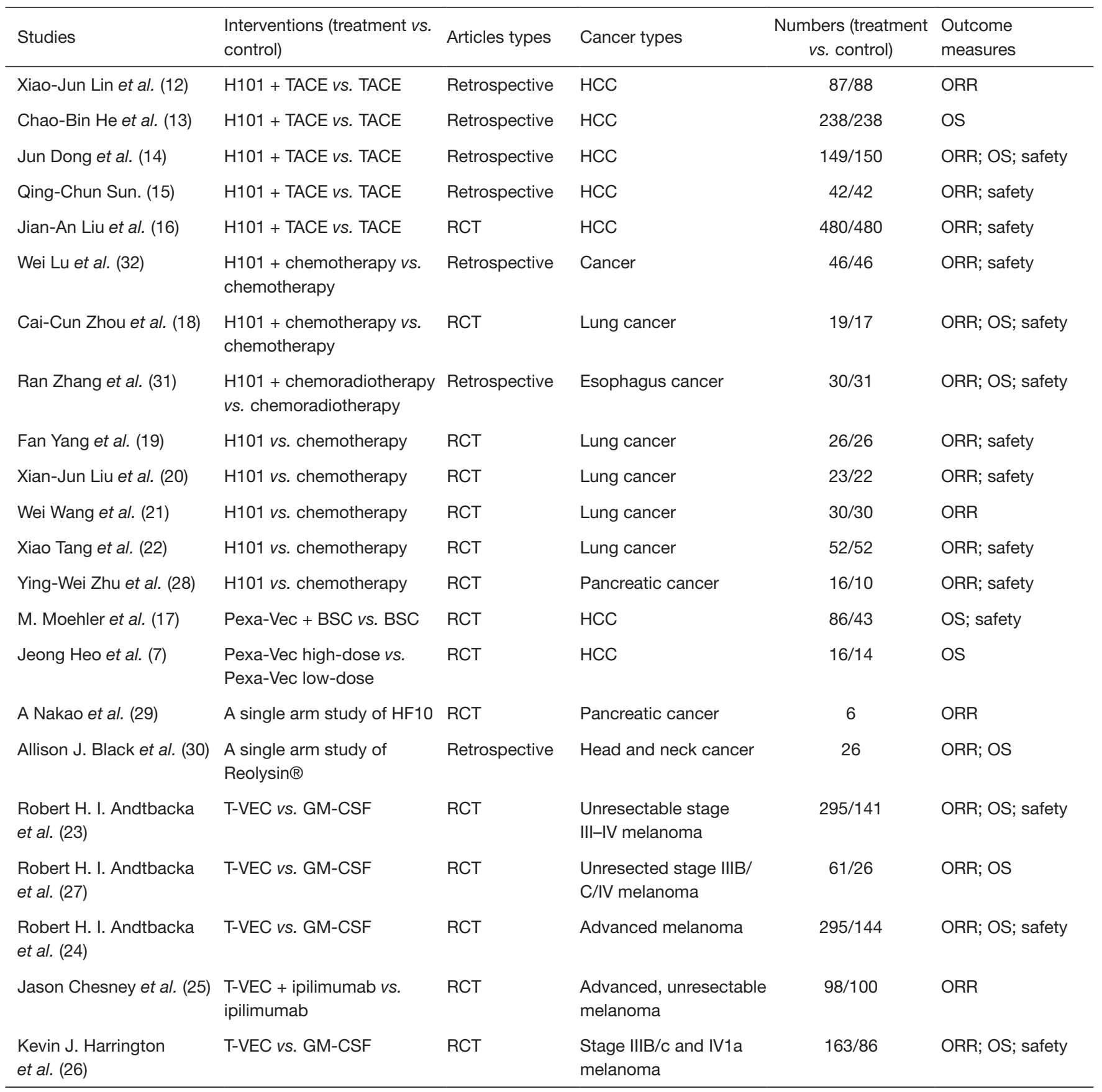

TACE, transhepatic arterial chemoembolization; HCC, hepatocellular carcinoma; OS, overall survival; RCT, randomized controlled trial; T-VEC, Talimogene laherparepvec; GM-CSF granulocyte-macrophage colony-stimulating factor; ORR, overall response rate. 


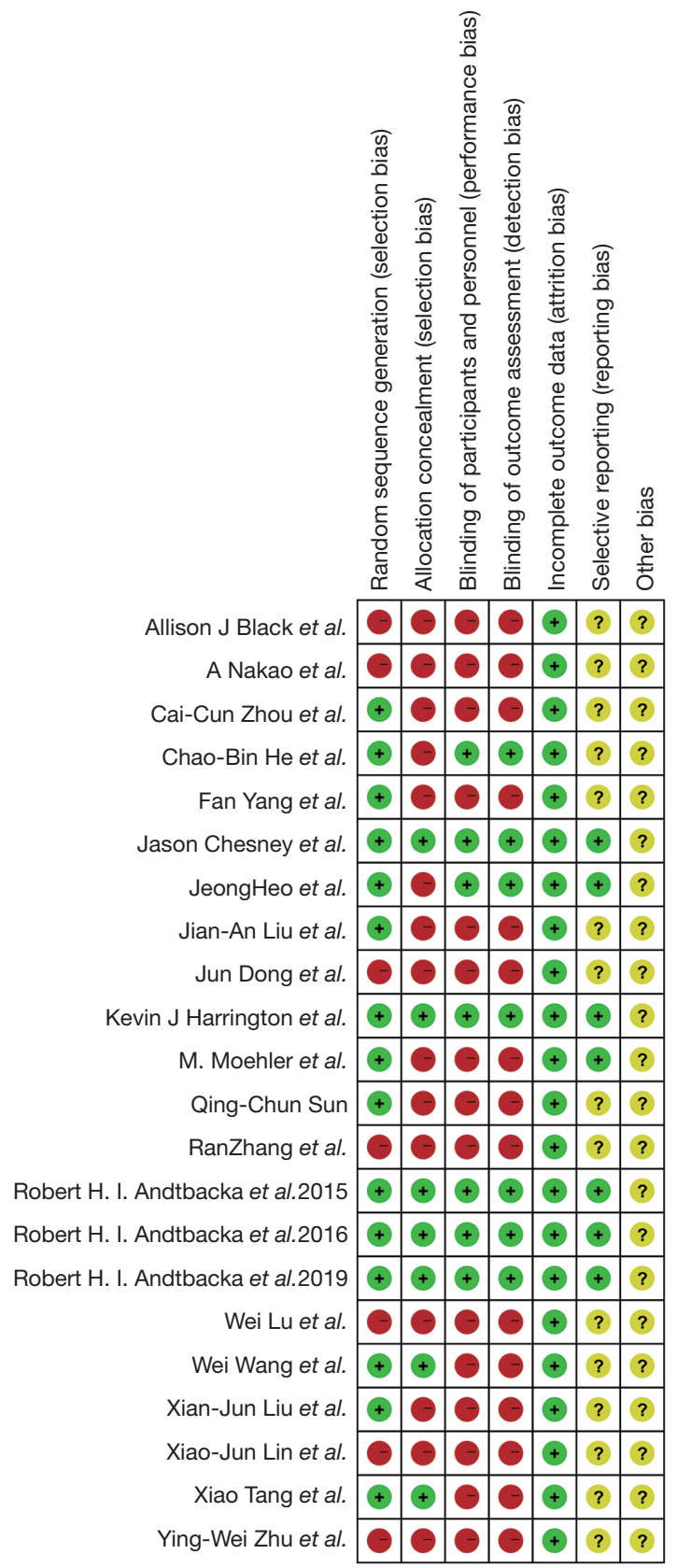

Figure 2 Authors' judgments about the risk of bias in the included studies.

chills, and pyrexia. The only grade 3 or $4 \mathrm{AE}$ occurring in $2 \%$ of T-VEC-treated patients was cellulitis (2.1\%) (23). The AEs of OVs in the treatment of intermediate to advanced solid tumors mainly included fever, nausea and vomiting, leukopenia, hypotension, etc. No serious complications were observed. The side effects of OVs were similar to those of traditional therapies. Therefore, OV has acceptable clinical safety in solid cancers.

\section{Sensitivity analysis}

Sensitivity analysis was also performed by sequentially removing individual studies to determine whether their exclusion resulted in a substantial change in the pooled estimates and heterogeneity. The pooled analysis of efficacy in the treatment of intermediate to advanced solid tumors was reliable and stable between both groups (Figure 8).

\section{Discussion}

In China, the incidence and mortality of liver cancer, lung cancer and esophageal cancer are relatively high. At present, traditional treatments of cancer, radiotherapy and chemotherapy usually cause severe side effects and cannot completely kill cancer cells. In recent years, OVs, a new type of antitumor drug, have gradually attracted people's attention due to their unique advantage of killing tumor cells specifically without damaging normal cells. H101 plays an important role in the antitumor field as the first approved OV drug. With numerous clinical trials, H101 has been demonstrated to have excellent efficacy and good safety in the treatment of solid tumors, especially in liver cancer and lung cancer. To date, T-VEC intrahepatic injection in combination with intravenous pembrolizumab at standard doses in patients with HCC or liver metastases has been demonstrated to be feasible and tolerable (33). Most clinical trials of OVs are still under research and development.

In this study, 22 studies were included to explore the efficacy and safety of OVs in the treatment of intermediate to advanced solid tumors. The results showed that OVs could improve the efficacy and prolong the survival time of patients. The common adverse reactions among patients in the OV group were fever, vomiting, leukopenia, etc., without serious AEs. Compared with that of chemotherapy alone, the efficacy of H101 monotherapy or H101 in combination with chemotherapy for solid tumors was significantly better. Zhang et al. (31) studied 87 patients and found that the efficacy, OS rate (1-, 2- and 3-year), median OS and median PFS were significantly higher in the H101 combined with chemotherapy group and were superior to chemotherapy monotherapy in the treatment of tumors. Moreover, the incidence of adverse reactions was similar. The ORR of patients in the H101 treatment group was significantly higher than that in the chemotherapy alone 


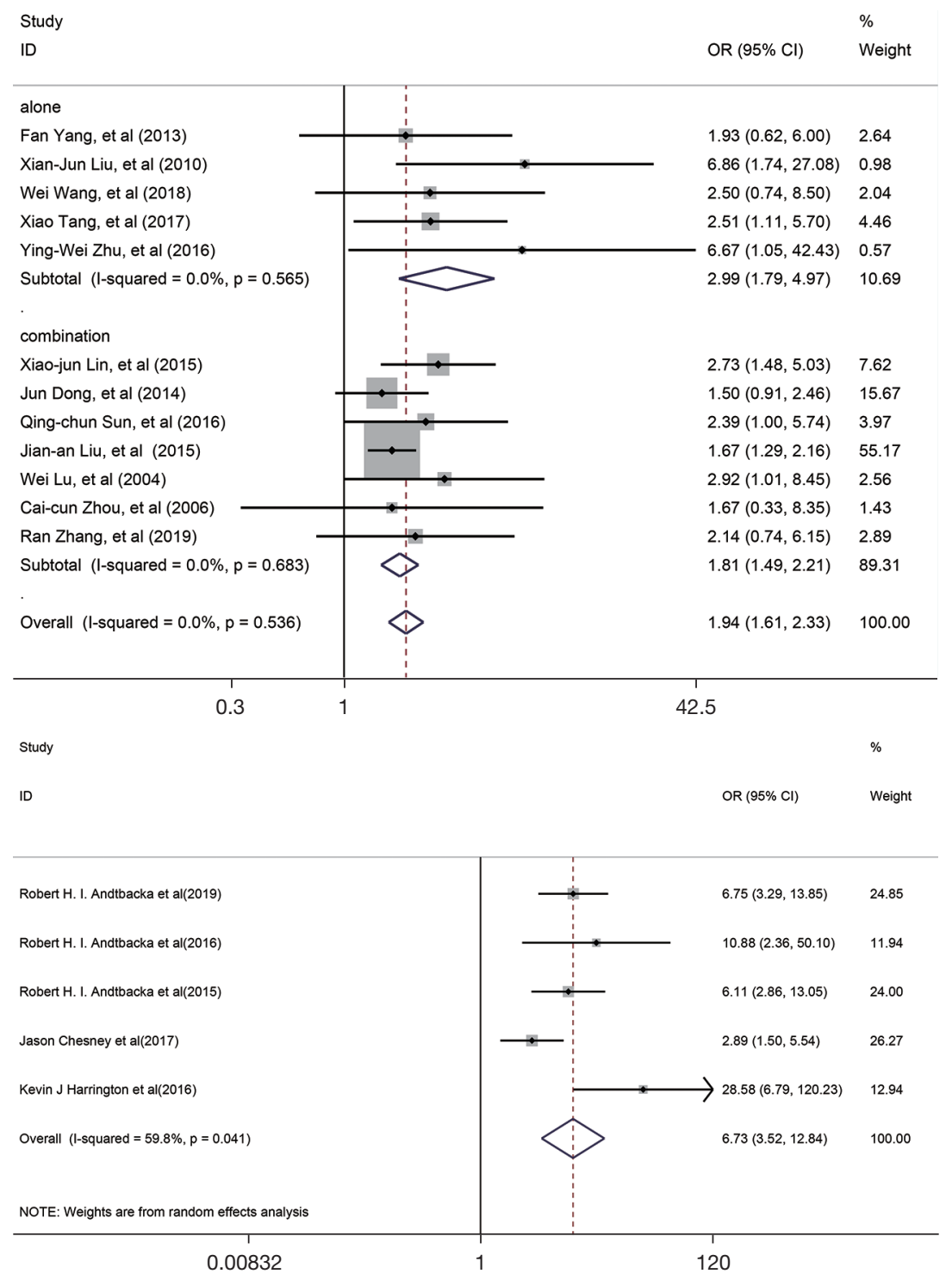

Figure 3 Forest map of the ORRs between the H101 and T-VEC groups. ORR, objective response rate; OR, odds ratio; CI, confidence interval.

group, and H101 prolonged patient OS. H101 has a good therapeutic effect and yields a good prognosis for patients. In this systematic review, the subgroup analysis showed that the incidence of fever in the H101 group was significantly higher than that in the chemotherapy group. Other adverse reactions, such as nausea, vomiting, leukopenia, bone marrow suppression, etc. were similar between H101 and chemotherapy alone, which indicated that OVs have good tolerance. Moreover, fever can promote the body's own resistance to disease, induce autoimmune ability, accelerate antitumor immunity and enhance antitumor effects.
T-VEC was well tolerated and resulted in a higher DRR $(\mathrm{P}<0.001)$ and longer median OS $(\mathrm{P}=0.051)$, particularly in untreated patients or those with stage IIIB, IIIC, or IVM1a disease (23). Administration of talimogene laherparepvec was associated with an improved ORR, DRR, and OS (24).

Additionally, the OS rates, median OS time, disease control rate (DCR) and mortality were analyzed by various types of studies. Four studies (12-14,31) proved that the 1-, 2-, and 3-year OS rates in the H101 group were higher than those in the chemotherapy group. As displayed in Table 2, the 1-, 2-, and 3-year OS rates in the H101 group 


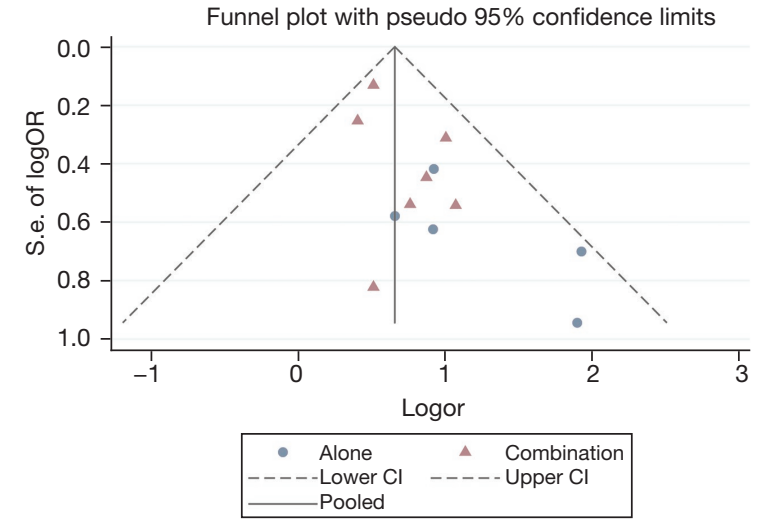

Figure 4 Forest map of OS between the H101 and T-VEC groups. OS, overall survival; CI, confidence interval. ranged from $61.3 \%$ to $87.5 \%, 35.6 \%$ to $60 \%$ and $29.5 \%$ to $40.5 \%$, respectively. Five studies assessed the effect of H101 on the median OS time $(12,13,18,21,22)$. All these studies reported improved effects on the median OS time after H101 intervention. Dong et al. (14) investigated the effect of H101 on the DCR but found no significant results. Zhu et al. (28) suggested that treatment with H101 significantly reduced mortality compared with chemotherapy.

In terms of other OVs, only one study by treatment with Reolysin reported that the ORR was $26.9 \%$ for head and neck cancer (30). A randomized multicenter Phase IIb trial (TRAVERSE) of Pexa-Vec revealed that PexaVec plus Best Supportive Care (BSC) did not improve OS compared with BSC alone. The median OS was 4.2 months

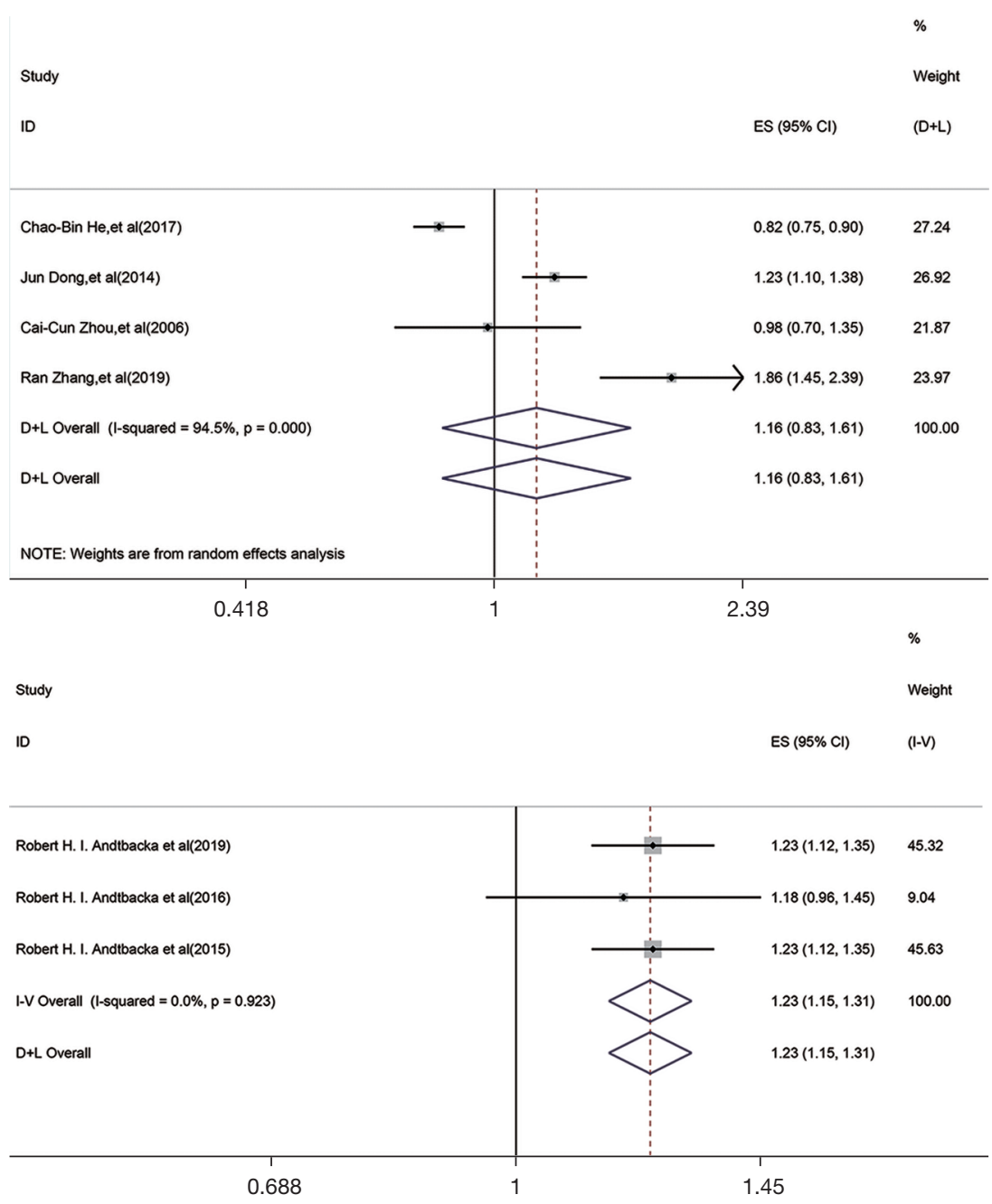

Figure 5 Forest map of OS between the H101 and T-VEC groups. OS, overall survival; ES, effect size; CI, confidence interval. 


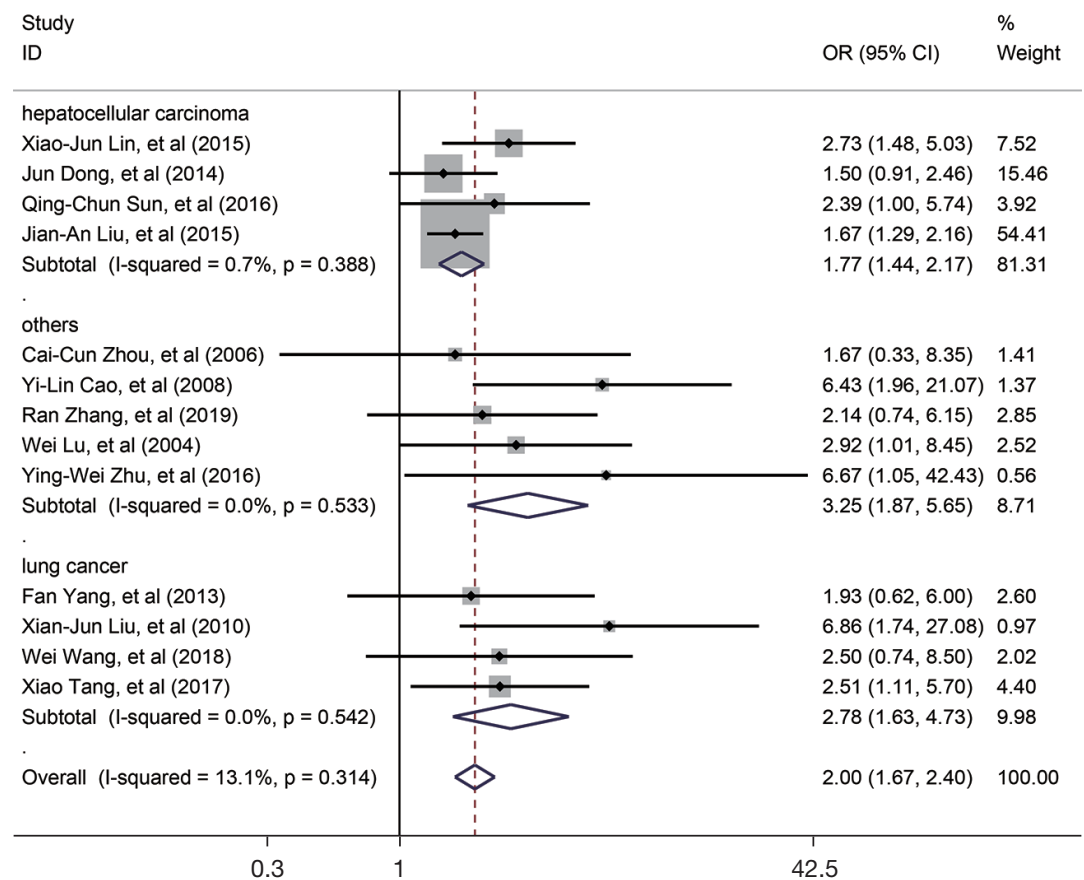

Figure 6 The OR of different H101-treated cancer types. OR, odds ratio; CI, confidence interval.

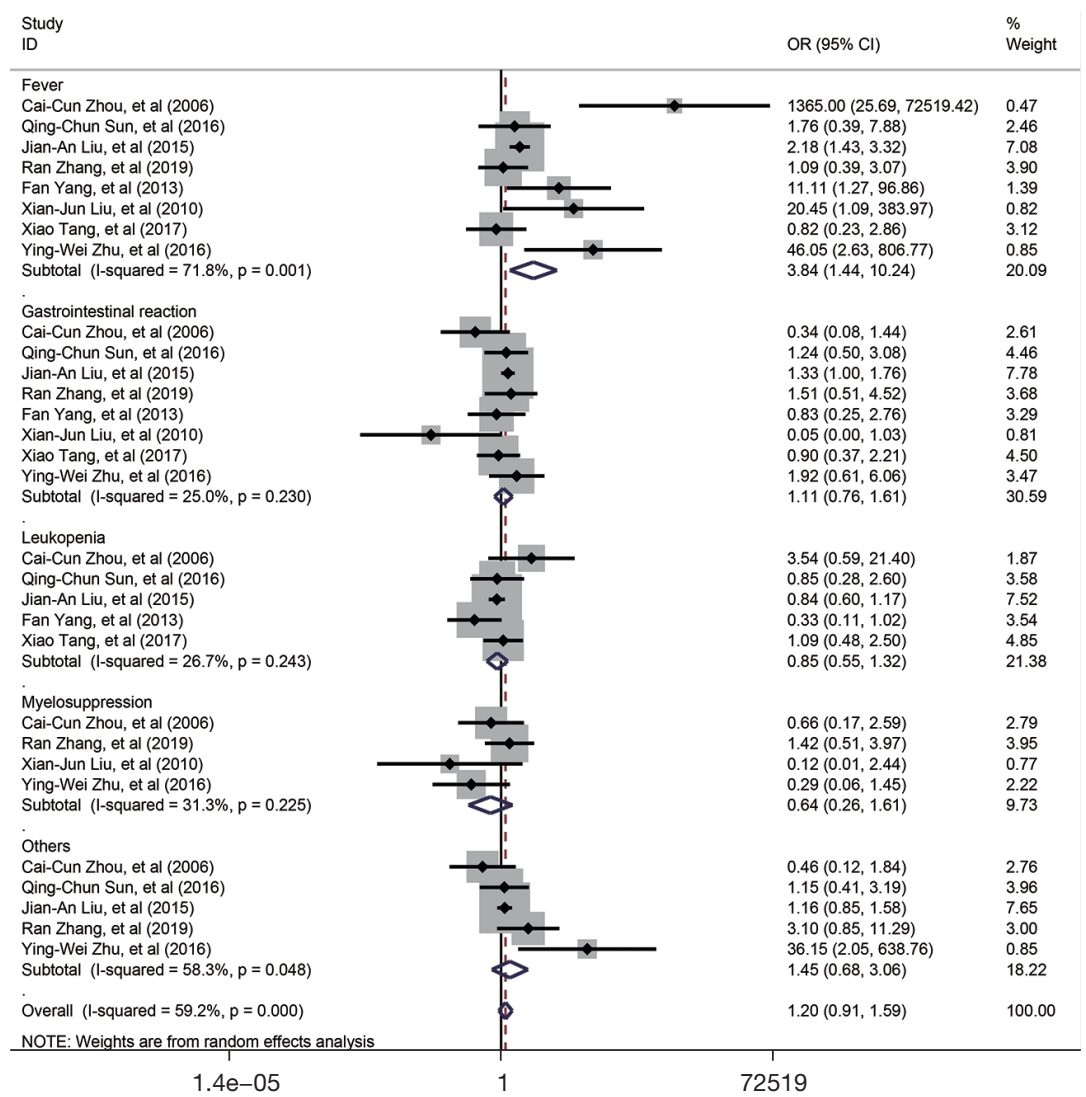

Figure 7 Forest map of adverse reaction rates between the H101 and chemotherapy groups. OR, odds ratio; CI, confidence interval. 


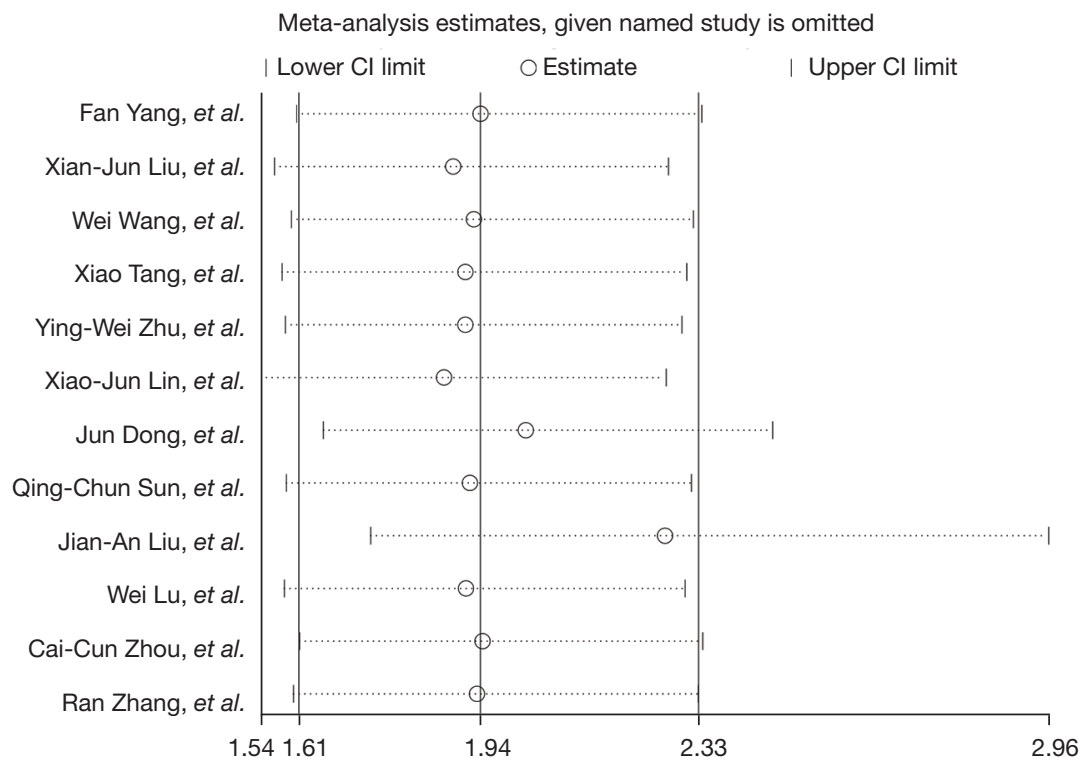

Figure 8 Sensitivity analysis of efficacy between the H101 and chemotherapy groups. CI, confidence interval.

for Pexa-Vec combined with BSC and 4.4 months for BSC alone. However, a randomized Phase 2 dose-finding trial of Pexa-Vec indicated that subject survival was significantly associated with the dose of Pexa-Vec (median survival of 14.1 months on the high dose compared to 6.7 months on the low dose). There was no significant difference in TTP between Pexa-Vec combined with BSC and BSC alone (17). A phase I study in six patients concluded that after treatment with HF10 OV, two patients were classified as progressive disease, three patients as stable disease and one patient as partial response according to modified RECIST criteria. The serological tumor marker CA19-9 was decreased in three patients. Thus, HF10 had some therapeutic potential. Early reports from a phase Ib clinical trial of T-VEC and ipilimumab suggest a response rate of $56 \%$ and a CR rate of $33 \%$; a randomized phase II study is ongoing (34). The meta-analysis had the following limitations. First, the study did not focus on one tumor due to insufficient articles to analyze the efficacy and safety of OVs, which may increase heterogeneity. Second, the sample size was small, and most of the studies did not describe the random methods, allocation concealment or blinding methods. Therefore, the methodology was not of high quality. Third, except for H101, studies of other OVs are few. Heterogeneity may be affected by disease type or virus type. Therefore, the above conclusions indicated that there is a high probability of publication bias. However, this systematic evaluation showed that OVs have significant effects and good safety for cancers. However, to obtain a more reliable conclusion, large-sample and multicenter RCTs are still needed.

On the basis of the meta-analysis, OVs have a significant antitumor effect on cancers and are superior to traditional treatment. Many of the adverse reactions are fever and vomiting, with good safety and no serious side effects. However, more high-quality studies are needed to verify this hypothesis. By equipping OVs with functional transgenes, a complete set of OVs will have multiple antitumor functions in the future, and appropriate virus combinations can be selected according to the type and stage of cancer.

\section{Conclusions}

* The ORR in patients treated with H101 or T-VEC was significantly higher than that in the control group.

* Compared with the control group, the H101 or T-VEC group had notably prolonged OS.

* Patients treated with H101 or T-VEC had significantly reduced mortality compared with those treated with chemotherapy.

* The overall incidence of treatment-related adverse effects was similar between the H101 group and chemotherapy group.

* The AEs associated with OVs in the treatment of intermediate to advanced solid tumors mainly included fever, nausea and vomiting, leukopenia, hypotension, etc. 
Table 2 The therapeutic effects of OVs based on included studies

\begin{tabular}{|c|c|c|c|}
\hline Studies & \multicolumn{2}{|c|}{ Survival benefits } & $P$ value \\
\hline Xiao-Jun Lin et al. (12) & $\begin{array}{l}\text { median OS time }=12.8 \text { months }(12.95 \\
\pm 8.36 \text { months); the OS rates at } 1 \text { and } 2 \text { years } \\
\text { were } 69 \% \text { and } 60 \% ; \text { PFS }=10.49 \text { months; } \\
\text { ORR }=60.9 \%\end{array}$ & $\begin{array}{l}\text { median OS time }=11.6 \text { months }(12.87 \\
\pm 8.28 \text { months); The OS rates at } 1 \text { and } 2 \text { years } \\
\text { were } 60 \text { and } 44 \% ; \text { PFS }=9.72 \text { months; ORR } \\
=36.4 \%\end{array}$ & All $P<0.05$ \\
\hline Jun Dong et al. (14) & $\begin{array}{l}\text { Mean OS }=1,526 \text { d }(95 \% \text { Cl: } 1,365.7- \\
1,685.9 \text { d); } 1-, 2-\text { and } 3-\text { year OS rates were } \\
61.7 \%, 35.6 \%, 29.5 \% \text {; ORR }=73.8 \% \text {; DCR } \\
=75.8 \%\end{array}$ & $\begin{array}{l}\text { Mean OS }=1,236 \text { d }(95 \% \text { Cl: } 939.6-1,531.8 \text { d); } 1 \text {-, } \\
\text { 2- and 3-year OS rates were } 54.0 \%, 30.0 \% \text {, } \\
\text { 21.3\%; ORR =65.3\%; DCR }=66.7 \%\end{array}$ & $\begin{array}{l}\text { OS } P<0.05 \\
\text { ORR, DCR } \\
P>0.05\end{array}$ \\
\hline Wei Lu et al. (32) & ORR $=30.4 \%$ & ORR $=13.0 \%$ & $\mathrm{P}<0.05$ \\
\hline Cai-Cun Zhou et al. (18) & ORR $=26.3 \%$ & ORR $=17.6 \%$ & - \\
\hline Ran Zhang et al. (31) & $\begin{array}{l}\text { Median OS =34.6 months; } 1 \text { - and 2-year OS } \\
\text { rates were } 87.5 \%, 58.3 \% \text {; median PFS } \\
=23.8 \text { months; ORR }=46.7 \%\end{array}$ & $\begin{array}{l}\text { Median OS =18.6 months; } 1 \text { - and 2-year OS } \\
\text { rates were } 61.5 \%, 26.9 \% \text {; median PFS } \\
=14.8 \text { months; ORR }=29 \%\end{array}$ & All $P<0.05$ \\
\hline Fan Yang et al. (19) & ORR $=69.23 \%$ & ORR $=53.84 \%$ & $\mathrm{P}<0.05$ \\
\hline Xian-Jun Liu et al. (20) & $\mathrm{ORR}=82.61 \%, \mathrm{CR}=47.83 \%$ & ORR $=40.91 \% ; C R=18.18 \%$ & All $\mathrm{P}<0.05$ \\
\hline Wei Wang et al. (21) & $\begin{array}{l}\text { ORR }=83.3 \% \text {; the average quality of life was } \\
\text { improved by } 15 \text { points }\end{array}$ & $\begin{array}{l}\text { ORR }=66.7 \% \text {; the average quality of life was } \\
\text { improved by } 5 \text { points }\end{array}$ & All $\mathrm{P}<0.05$ \\
\hline A Nakao et al. (29) & $\mathrm{PR}=1 ; \mathrm{SD}=3 ; \mathrm{PD}=2$ & & - \\
\hline Allison J. Black et al. (30) & ORR $=26.9 \%$; median OS =7.1 months & & - \\
\hline $\begin{array}{l}\text { Robert H. I. Andtbacka } \\
\text { et al. (23) }\end{array}$ & $\begin{array}{l}\text { ORR }=31.5 \% ; \text { median OS }=23.3 \\
\text { DRR }=19.0 \%\end{array}$ & ORR $=6.4 \% ;$ median OS =18.9; DRR =1.4\% & $\mathrm{P}<0.05$ \\
\hline $\begin{array}{l}\text { Robert H. I. Andtbacka } \\
\text { et al. (27) }\end{array}$ & $\begin{array}{l}\text { ORR }=47.5 \% ; \text { median OS }=29.7 \\
\text { DRR }=36.1 \%\end{array}$ & ORR $=7.7 \% ;$ median OS =25.2; DRR =3.8\% & $\mathrm{P}<0.05$ \\
\hline $\begin{array}{l}\text { Robert H. I. Andtbacka } \\
\text { et al. (24) }\end{array}$ & $\begin{array}{l}\text { ORR }=26.4 \% ; \text { median OS }=23.3 \\
\text { DRR }=16.3 \%\end{array}$ & ORR $=5.7 \% ;$ median OS =18.9; DRR =2.1\% & $\mathrm{P}<0.05$ \\
\hline Jason Chesney et al. (26) & ORR =39\%; & ORR $=18 \%$ & $\mathrm{P}<0.05$ \\
\hline $\begin{array}{l}\text { Kevin J. Harrington } \\
\text { et al. (25) }\end{array}$ & $\begin{array}{l}\text { ORR }=40.5 \% ; \text { median OS }=40.1 \\
\text { DRR }=25.2 \%\end{array}$ & ORR =2.3\%; median OS =21.5; DRR =1.2\% & $\mathrm{P}<0.05$ \\
\hline
\end{tabular}

PR, partial response; PD, progressive disease; OS, overall survival; PFS, progression free survival; DRR, durable response rate; ORR, overall response rate; AFP, serum a-fetoprotein; DCR, disease control rate; $\mathrm{Cl}$, confidence interval. 
* OVs have acceptable clinical safety in solid cancers and are superior to traditional treatment.

\section{Acknowledgments}

Funding: None.

\section{Footnote}

Reporting Checklist: The authors have completed the PRISMA reporting checklist. Available at https://dx.doi. org/10.21037/tcr-21-905

Conflicts of Interest: All authors have completed the ICMJE uniform disclosure form (available at https://dx.doi. org/10.21037/tcr-21-905). The authors have no conflicts of interest to declare.

Ethical Statement: The authors are accountable for all aspects of the work in ensuring that questions related to the accuracy or integrity of any part of the work are appropriately investigated and resolved.

Open Access Statement: This is an Open Access article distributed in accordance with the Creative Commons Attribution-NonCommercial-NoDerivs 4.0 International License (CC BY-NC-ND 4.0), which permits the noncommercial replication and distribution of the article with the strict proviso that no changes or edits are made and the original work is properly cited (including links to both the formal publication through the relevant DOI and the license). See: https://creativecommons.org/licenses/by-nc-nd/4.0/.

\section{References}

1. Siegel R, Ma J, Zou Z, et al. Cancer statistics, 2014. CA Cancer J Clin 2014;64:9-29.

2. Raja J, Ludwig JM, Gettinger SN, et al. Oncolytic virus immunotherapy: future prospects for oncology. J Immunother Cancer 2018;6:140.

3. Cheng PH, Wechman SL, McMasters KM, et al. Oncolytic Replication of E1b-Deleted Adenoviruses. Viruses 2015;7:5767-79.

4. Buonaguro FM, Tornesello ML, Izzo F, et al. Oncolytic virus therapies. Pharm Pat Anal 2012;1:621-7.

5. Kaufman HL, Kohlhapp FJ, Zloza A. Oncolytic viruses: a new class of immunotherapy drugs. Nat Rev Drug Discov 2015;14:642-62.
6. Marcato P, Shmulevitz M, Pan D, et al. Ras transformation mediates reovirus oncolysis by enhancing virus uncoating, particle infectivity, and apoptosis-dependent release. Mol Ther 2007;15:1522-30.

7. Heo J, Reid T, Ruo L, et al. Randomized dose-finding clinical trial of oncolytic immunotherapeutic vaccinia JX594 in liver cancer. Nat Med 2013;19:329-36.

8. Huang X, Jia R, Zhao X, et al. Recombinant oncolytic adenovirus H101 combined with siBCL2: cytotoxic effect on uveal melanoma cell lines. Br J Ophthalmol 2012;96:1331-8.

9. Hill C, Carlisle R. Achieving systemic delivery of oncolytic viruses. Expert Opin Drug Deliv 2019;16:607-20.

10. Alberts P, Tilgase A, Rasa A, et al. The advent of oncolytic virotherapy in oncology: The Rigvir ${ }^{\circledR}$ story. Eur J Pharmacol 2018;837:117-26.

11. Liu TC, Galanis E, Kirn D. Clinical trial results with oncolytic virotherapy: a century of promise, a decade of progress. Nat Clin Pract Oncol 2007;4:101-17.

12. Lin XJ, Li QJ, Lao XM, et al. Transarterial injection of recombinant human type-5 adenovirus H101 in combination with transarterial chemoembolization (TACE) improves overall and progressive-free survival in unresectable hepatocellular carcinoma (HCC). BMC Cancer 2015;15:707.

13. He CB, Lao XM, Lin XJ. Transarterial chemoembolization combined with recombinant human adenovirus type 5 H101 prolongs overall survival of patients with intermediate to advanced hepatocellular carcinoma: a prognostic nomogram study. Chin J Cancer 2017;36:59.

14. Dong J, Li W, Dong A, et al. Gene therapy for unresectable hepatocellular carcinoma using recombinant human adenovirus type 5. Med Oncol 2014;31:95.

15. Sun QC. Clinical study of recombinant human 5 type adenovirus injection in the interventional therapy of liver cancer. Chinese Community Dotors;32:23-4.

16. Liu JA, Yu J, Xu P. An investigation on the curative effect of human recombinant adenovirus type 5 injection on the interventional therapy of primary liver cancer. Electronic Journal of Clinical General Surgery 2015:43-7.

17. Moehler M, Heo J, Lee HC, et al. Vaccinia-based oncolytic immunotherapy Pexastimogene Devacirepvec in patients with advanced hepatocellular carcinoma after sorafenib failure: a randomized multicenter Phase IIb trial (TRAVERSE). Oncoimmunology 2019;8:1615817.

18. Zhou CC, Xu Y, Ni J, et al. Oncolytic adenovirus H101 in combination with vinorelbine/cisplatin chemotherapy for advanced non-small cell lung cancer. Tumor 
2006;026:613-7.

19. Yang F, Lu B, Hu CY, et al. Intrathoracic injection of recombinant human adenovirus type-5 for advanced lung cancer with malignant pleural effusion. The Journal of Practical Medicine 2013;29:2885-6.

20. Liu XJ, Peng JX, Lu JC, et al. Perfusion of recombinant human adenovirus type 5 injection in pleural cavity to patients with lung cancer combining malignant pleural effusions. Journal of Clinical Pulmonary Medicine 2010;015:1539-40.

21. Wang W, Li FF, Xiao CZ, et al. Therapeutic effects analysis of Lung Cancer Malignant Pleural Effusion by GongAi LiShui Powder for External Application in combination with recombinant human type-5 adenovirus H101. Chinese Journal of Gerontology 2018;038:5184-6.

22. Tao C, Wang GH, Wang WM. Analysis of effect by human recombinant type 5 adenovirus through intrapleural injection in the treatment of advanced lung cancer malignant pleural effusion. Chinese Journal of Modern Drug Application 2017;11:91-3.

23. Andtbacka RH, Kaufman HL, Collichio F, et al. Talimogene Laherparepvec Improves Durable Response Rate in Patients With Advanced Melanoma. J Clin Oncol 2015;33:2780-8.

24. Andtbacka RH, Agarwala SS, Ollila DW, et al. Cutaneous head and neck melanoma in OPTiM, a randomized phase 3 trial of talimogene laherparepvec versus granulocytemacrophage colony-stimulating factor for the treatment of unresected stage IIIB/IIIC/IV melanoma. Head Neck 2016;38:1752-8.

25. Chesney J, Puzanov I, Collichio F, et al. Randomized, Open-Label Phase II Study Evaluating the Efficacy and Safety of Talimogene Laherparepvec in Combination With Ipilimumab Versus Ipilimumab Alone in Patients With Advanced, Unresectable Melanoma. J Clin Oncol 2018;36:1658-67.

26. Harrington KJ, Andtbacka RH, Collichio F, et al. Efficacy and safety of talimogene laherparepvec versus granulocyte-macrophage colony-stimulating factor

Cite this article as: Gao P, Ding G, Wang L. The efficacy and safety of oncolytic viruses in the treatment of intermediate to advanced solid tumors: a systematic review and meta-analysis. Transl Cancer Res 2021;10(10):4290-4302. doi: 10.21037/tcr-21-905 in patients with stage IIIB/C and IVM1a melanoma: subanalysis of the Phase III OPTiM trial. Onco Targets Ther 2016;9:7081-93.

27. Andtbacka RHI, Collichio F, Harrington KJ, et al. Final analyses of OPTiM: a randomized phase III trial of talimogene laherparepvec versus granulocyte-macrophage colony-stimulating factor in unresectable stage III-IV melanoma. J Immunother Cancer 2019;7:145.

28. Zhu YW, Gong L, Wu GY, et al. Therapeutic effects analysis of tumor injection of H101 guided by endoscopic ultrasonography for patients with pancreatic carcinoma. Journal of Nanjing Medical University 2016;36:1166-9.

29. Nakao A, Kasuya H, Sahin TT, et al. A phase I doseescalation clinical trial of intraoperative direct intratumoral injection of HF10 oncolytic virus in non-resectable patients with advanced pancreatic cancer. Cancer Gene Ther 2011;18:167-75.

30. Black AJ, Morris DG. Clinical trials involving the oncolytic virus, reovirus: ready for prime time? Expert Rev Clin Pharmacol 2012;5:517-20.

31. Zhang R, Li WN, Lv S, et al. Clinical Efficacy of Endoscopic Injection of Recombinant Human Adenovirus Type 5 for Advanced Esophageal Cancer. Chinese Journal of Gastroenterology 2019;24:30-4.

32. Lu W, Zheng S, Li XF, et al. Intra-tumor injection of H101, a recombinant adenovirus, in combination with chemotherapy in patients with advanced cancers: a pilot phase II clinical trial. World J Gastroenterol 2004;10:3634-8.

33. Hecht JR, Pless M, Cubillo A, et al. Early safety from a phase I, multicenter, open-label clinical trial of talimogene laherparepvec (T-VEC) injected (inj) into liver tumors in combination with pembrolizumab (pem). J Clin Oncol 2020;38:3015.

34. Andtbacka RH, Ross M, Puzanov I, et al. Patterns of Clinical Response with Talimogene Laherparepvec (T-VEC) in Patients with Melanoma Treated in the OPTiM Phase III Clinical Trial. Ann Surg Oncol 2016;23:4169-77. 